\title{
Fault Analysis of AC Uninterruptible Power Supply System in Gas Pipeline Compressor Station
}

\author{
Chen Hanlin \\ China Petroleum Engineering \& Construction Corporation \\ Beijing, China \\ chenhanlin@cpecc.com.cn
}

\begin{abstract}
Regarding the AC UPS fault accident happened in one compressor station which caused severe consequence with gas transmission pipeline, this paper lists the possible reasons based on the recorded fault code and equipment configuration, and solutions are proposed for modification. In the end, recommendations are given for the future project design of $\mathrm{AC}$ UPS.
\end{abstract}

Keywords- Uninterruptible Power Supply; UPS; Gas Pipeline; Island Operation; Compressor Station; High Frequency; Power Frequency; Power Electronics; IGBT; Power Quality

\section{INTRODUCTION}

The purpose of a compressor station is to boost the pressure in a natural gas pipeline and move the natural gas further downstream. This compressor station is one of the middle compressor stations along an international long distance gas pipeline. In this compressor station, gas turbine serves to operate the centrifugal compressor and gas engine generators are used as main power supply (total 3 nos., each is $1000 \mathrm{~kW}, 380 \mathrm{~V}, 50 \mathrm{~Hz}, 0.8 \mathrm{pf})$. The electrical system is of T-N-S type.

As per category of electrical loads, most power consuming devices are fed by normal and emergency bus bar separately, and important equipment such as Station Control System (SCS) and telecommunication system are powered by Uninterruptible Power Supply. Based on history record of Electrical Control System (ECS), gas generator \#3 is the only running generator with output power $600 \mathrm{~kW}$ during normal condition.

According to the official report from operators, the accident of AC UPS output flash-stop happened during hotstandby gas generator \#2 increasing power output after gas generator \#3 shutting down from one failure. The output of AC UPS suddenly stopped and automatically recovered after a very short time. The fail-safe type ESD (emergency shutdown) system which is powered by AC UPS was triggered by this flash-stop accident, and all running process equipments which connected with the SCS \& ESD system were emergency stopped. Besides, the communication between the compressor station and the pipeline SCADA central control station broke down by power failure.

The fault analysis will be carried out from the structure of AC UPS.

\section{STRUCTURE OF AC UPS}

The static uninterruptible power supply system (UPS) is of dual redundant configuration, normally both inverters shall be synchronized with each other and with the AC power input. The two inverters shall be operating in parallel and sharing the load equally. When a disturbance/fault occurs in any of the inverters, the faulty unit shall be automatically disconnected and the entire load supplied from the remaining inverter. If both inverters develop a fault, the load shall be transferred to the bypass supply through the static switch.

Refer to Figure.1 for the single line diagram of AC UPS system.

According to the manufacture specification, the incoming cables for UPS main unit \#1 and \#2 are 3C+PE type, and the incoming cable for bypass unit is $4 \mathrm{C}+\mathrm{PE}$ type. The isolation transformers for UPS main unit \#1 and \#2 are of delta-delta winding, and the bypass transformer is of star-star winding. Nominal output voltage for DC battery bank is 720VDC with 3 wires (positive, neutral, negative). IGBT is used in invertor unit.

\section{FAULT RECORD OF AC UPS}

As per the report, fault codes record from the mimic panel of UPS \#1 and \#2 were same during the flash-stop accident, refer to Table 1.

According to the alarm code, during the flash-stop moment, both invertor units of UPS \#1 and \#2 were shut down and bypass was not available. Beside, some internal error was found by the self-exam module. Because AC UPS automatically and immediately recovered and none hardware fault was found after later examination, it is obvious that the transition period of generator \#2 rapid increasing power output caused this accident.

TABLE I. ALARM CODE DESCRIPTION FOR THE FLASH-STOP ACCIDENT

\begin{tabular}{|l|c|c|}
\hline Code & Alarm Name & Description \\
\hline A15 & Bypass fault & Bypass is not available \\
\hline A25 & Invertor closed & Invertor is shut down \\
\hline A28 & UPS self-exam fault & System warning \\
\hline
\end{tabular}




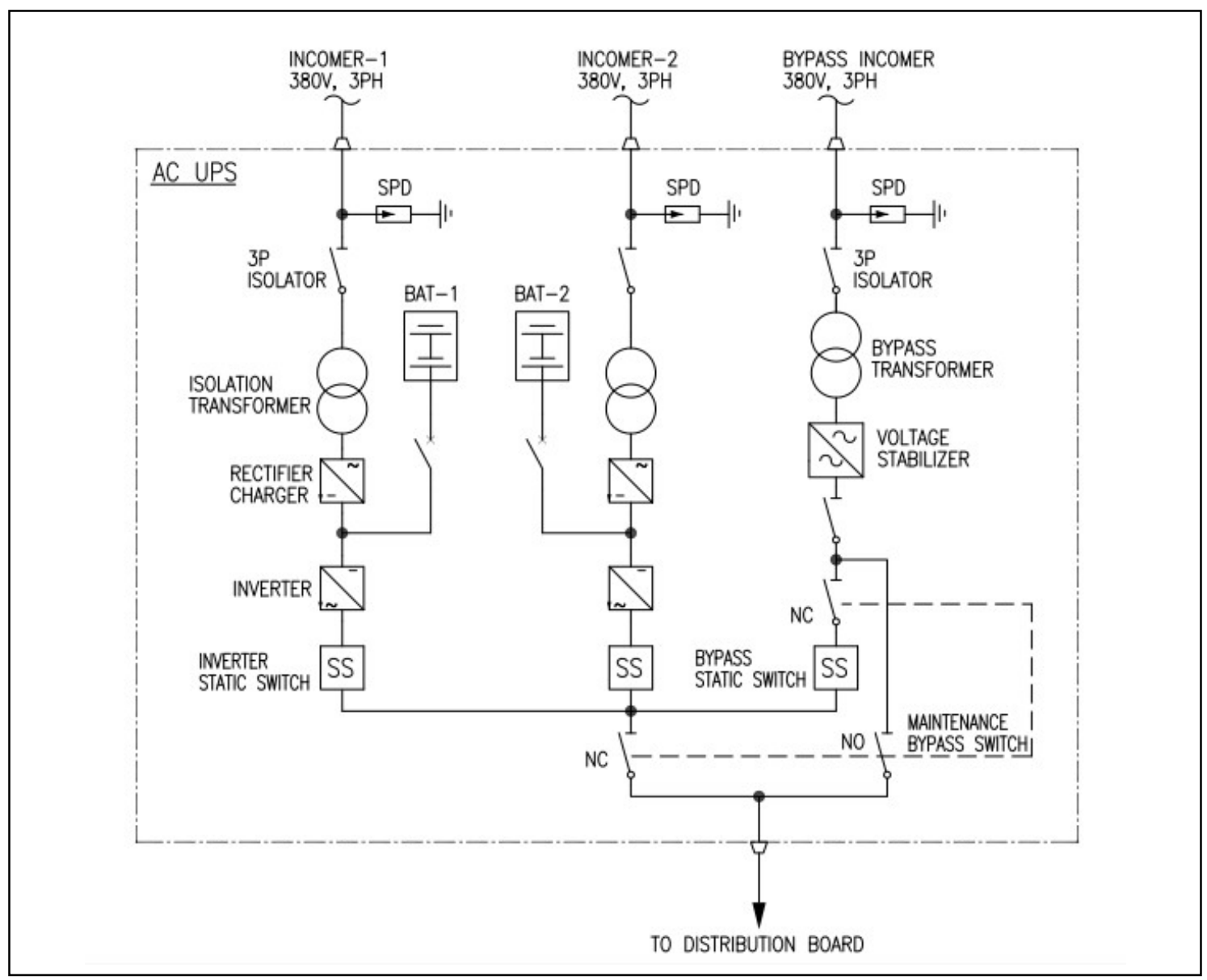

Figure 1. Single Line Diagram of AC UPS system

\section{FAULT ANALYSIS}

Based on the universal practice of factory fabrication, there are one common neutral bus bar and one common earthing bus bar inside the AC UPS cabinets, which means that all neutral conductor belong the AC UPS system will be bonded to the common neutral bus bar, and all earthing conductor including cabinets' enclosure will be connected to the common earthing bus bar. The 'common' neutral provides zero-potential reference for the AC UPS working and the 'common' earthing provides electrical equipotential bonding for protection.

The Y-winding neutral point of bypass transformer and the neutral wire of DC battery bank are bonded to the common neutral bus bar as well. During the transient period, the electrical load of gas generator was increasing rapidly and causing turbulence to this small power system of island operation. The feedback harmonics and unbalanced current between phase lines made the power quality very poor. The transient current in the system neutral line was delivered to the AC UPS system via the Y-winding neutral point of the bypass transformer unit. The interfere from the power system flowed into the common neutral bus bar of AC UPS system, interfered with the DC neutral line and caused the control circuit shutting down the invertors of UPS \#1 and \#2 together. Besides, it's also possible that the harmonics from the power system interfered with the control circuit of AC UPS and generated incorrect order. After all the interfere disappeared, the UPS system automatically recovered.

It's clear that the disturbance from the power system neutral line caused the accident.

\section{SOLUTION}

Since the interfere from the system caused the accident, then it is reasonable by segregating the system neutral conductor from the AC UPS neutral bus bar to solve this problem.

It is applicable to replace the bypass transformer of $\mathrm{Y}-\mathrm{Y}$ winding to $\mathrm{D}-\mathrm{Y}$ winding to make a 'floating' neutral point inside the AC UPS system. Then the AC UPS common neutral will be isolated from the system neutral, and the disturbance in the system neutral conductor will not affect the AC UPS. The AC UPS output neutral line will be related with the DC battery bank neutral wire only.

This solution is the most suitable and economic way to prevent the similar accident happening again. No more 
similar accident was reported from this compressor station after this modification. Meanwhile, for future brand new project, it is better to consider the feature of island operation power system and design the AC UPS with better configuration at the beginning.

\section{RECOMMENDATION FOR FUTURE PROJECT}

For future similar project design of AC UPS system, it is necessary to consider the following suggestions:

- Neutral zero-potential point should not be involved in IGBT inverter working, which means the interconnection power cable between DC battery and UPS main unit should be 2-core type (positive, negative).

- The built-in neutral bus of AC UPS should be isolated from power system neutral conductor.

- Input isolation transformer should be considered in UPS main unit and bypass unit, and Y winding is not recommended for primary winding. Moreover, output isolation transformers should be configured for inverter as well.

- Special measures and equipment should be considered to improve the power quality of island operation system.
The stability and performance of AC UPS system will be better after isolating the system interfere and optimizing the electrical system power quality.

\section{CONCLUSION}

For the island operation power system of gas compressor station, the power quality is not very good based on the character of power load and scale of the power distribution network. The 'common' practice of equipment configuration might not be suitable for the small power system, where harmonics, unbalanced current, voltage fluctuation, power source stability appears frequently. Hence, special cautions should be taken into account for the delicate power electronic devices, like the AC UPS system.

\section{REFERENCES}

[1] Wang Zhaoan, Huang Jun, "Technology of Power Electronics", Edition $4^{\text {th }}$, China Machine Press, 2001.

[2] IEC 62040, "Uniterruptible Power Supply (UPS)".

[3] IEEE Std 519-1992, "IEEE Recommendaed Practices and Requirements for Harmonic Control in Electrical Power Systems”.

[4] IEEE Std 1100-2005, "IEEE Recommended Practice for Powering and Grounding Electronic Equipment”.

[5] BS 7671-2008, "Requirements for Electrical Installations". 